\title{
A Survey on Credit-Based Incentive Schemes in Delay Tolerant Network
}

\author{
${ }^{1}$ Rajesaheb Kadam, ${ }^{2}$ Manoj Bangare \\ ${ }^{1}$ Research Scholar, Department of Information Technology, Savitribai Phule Pune University, Pune-411041, India \\ ${ }^{2}$ Assistance Professor, Department of Information Technology, Savitribai Phule Pune University, Pune-411041, India
}

\begin{abstract}
A Delay Tolerant Network is a network of regional networks having characteristics like long or variable delay, intermittent connectivity, asymmetric data rate and high error rates. Due to these characteristics, DTN is designed to operate in wide-ranging applications where end to end connectivity is not always guaranteed like space communication and inter-planetary communication. DTN uses the store-carry-and-forward mechanism to relay bundles from source node to the destination node. In DTN bundles are routed to destination node with the hypothesis that each intermediate node is willing to relay incoming bundle to next hop. In real time application, DTN nodes are controlled and handled by rational entities such as organisation so DTN nodes will behave selfishly and violates the hypothesis. To address the problem of selfish behaviour of node in DTN, various credit based incentive schemes are proposed with suggestion of some incentive should be offered to intermediate nodes so that they will participate in bundle relaying and nodes will not behave selfishly. In this paper, we surveyed and compared existing credit based incentive mechanisms. Also, stated some open research issues in DTN at last in conclusion.
\end{abstract}

Keywords: Delay Tolerant Network, Selfish Node, Selfishness

\section{Introduction}

A Delay Tolerant Network is a network of regional networks. It resides on top of regional networks and internet is also part of DTN. DTNs support interoperability of the networks which covers it by including long delays between and within regional networks, and by translating between regional network communications characteristics. DakNet project of India is a typical example of DTN [1]. In providing these functions, DTNs accommodate the mobility and limited power of evolving wireless communication devices. The DTN technology has wider scope; it includes radio frequency (RF), ultra wide band (UWB), free space optical and ultrasonic technologies [2]. Several networks including the internet also may not have assumptions which Delay Tolerant Network has. Characteristic of Delay Tolerant Networks are explained below:

Intermittent Connectivity: In a circumstance that, if there is no end to end connectivity persist then a network will divide into one or many parts, this is called Network Partitioning and in this condition TCP/IP-traditional protocols will not work.

Long or Variable Delay: Apart from occasional connectivity, variable delays at nodes and propagation delays between peers contribute to peer to peer delays that may compete for various applications and Internet protocols which depend on the quick return of acknowledgements.

Asymmetric Data Rates: many users with cable TV they uses Internet supports asymmetric nature of full duplex communication links which has bi-directional data rate. But if asymmetric nature is full, they can compete the traditional protocols.

High Error Rates: As the communication links are highly asymmetric bit errors on communication links must be recognized and must have to correct (which demands extra parity bits and more processing power ) or retransmission of the sent packet messages (which causes more network traffic). Some retransmissions of entire packets are needed including node to node other than end to end retransmission.

\subsection{Store-Carry-and-Forward mechanism in DTN}

DTNs removes the problems including high error rates, intermittent connectivity, asymmetric data rates, variable delays by using store and forward message switching mechanism. This is an old method, which is used in ancient times like postal systems in human society. Entire messages or fragments of application data are forwarded from a storage place to one site to another node which has storage capacity, followed by a path which reaches to the destination. Today's voicemail and email systems use Store and forwarding mechanism though these systems are not only include one-way cover but also has star communication, central storage device is independently contacted by the source and destination. The various storage places for example hard disk long lastingly hold messages. This storage is also called as a persistent storage; memory chips have characteristics of caching a data for a very short time. Routers in the network store and persist the packets or queued the packets for a very short time may be a milliseconds use memory chips. When they are looking and waiting for their next router routing table lookup and search for outgoing router port. Routers which are used in the Delay Tolerant Network need persistent storage for their queuing of data packets for a long time for the reasons as below: A communication link to the next hop may not be available for a long time. One node in a communicating pair may send or receive data much faster or more reliably than the other node. Message retransmission is needed which is transmitted before when any error occurs at an upstream 


\section{International Journal of Science and Research (IJSR) \\ ISSN (Online): 2319-7064}

Index Copernicus Value (2013): 6.14 | Impact Factor (2015): 6.391

node or link which is towards the destination, or if the upstream node decided to reject the forwarded packets. Single transfer moving of entire packets of packet the message switching technique is used which provides knowledge of the size of messages by the nodes of the network. Therefore intermediate storage space and retransmission bandwidth is very essential in such type of networks.

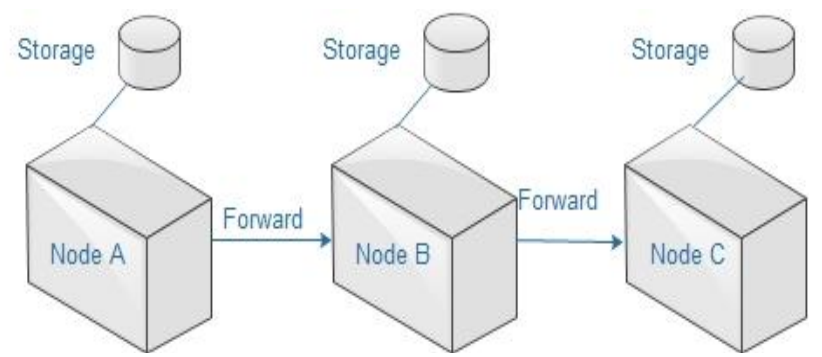

Figure 1: Store and Forward Mechanism in DTN

\section{$1.2 \quad$ DTN Specific Stack}

The unit of data exchange in a DTN is a bundle. A DTN node is a node with a bundle layer. It may be a host, or gateway, router acting as a source or destination. Traditional network use a TCP and IP protocol. In a DTN, the bundle layer is present below the application layer and hides the region specific network such as the internet, as depicted in Figure.

A network-specific layer is used below the bundle layer to interface with each different network layer protocol used. The Bundle Protocol, defined in RFC 5050 [3] and implements a bundle layer in the DTN architecture defined in RFC 4838 [4]. The Bundle Protocol supports and provides six classes of service $(\mathrm{CoS})$ for a bundle, such as Custody Transfer, Return Receipt, Bundle-Forwarding Notification, Custody-Transfer Notification, Authentication and Priority of Delivery (bulk, normal, expedited).

In DTNs, forwarding nodes such as routers and gateways need to be authenticated. The sender information is authenticated by intermittent nodes so that network resources will be conserved by preventing the relaying of prohibited traffic at the earliest opportunity. The unique property of the bundle layer is that support for in-transit storage with buffer. Bundles received from a sender can be stored in an intermediate node for the large amount of time; it may be minutes, hours, or even days. This store operation is performed by the network stack, at the bundle layer and transparently to the application layer. The intransit storage is the means to overcome the delays induced while a bundle moves node by node to its final destination, to avoid costly end-to-end retransmissions due to errors and timeout and allow exchange of data between two nodes that have no end to end communication path at any given time moment. The bundle protocol has a custody operation which allows an intermediate node to handle bundle delivery to the destination on behalf of distant senders.

\begin{tabular}{|c|}
\hline Application Layer \\
\hline Transport Layer \\
\hline Network Layer \\
\hline Data Link Layer \\
\hline Physical Layer \\
\hline
\end{tabular}

TCP/IP Stack

\begin{tabular}{|c|}
\hline Application Layer \\
\hline Bundle Layer \\
\hline Transport Layer \\
\hline Network Layer \\
\hline Data Link Layer \\
\hline Physical Layer \\
\hline
\end{tabular}

\section{DTN Protocol Stack}

Figure 2: DTN Specific Stack

In this paper, we surveyed the credit based incentive schemes and selfish behaviour of the nodes. The remaining paper is organized as follows. In Section 2 we briefly introduce the selfish behaviour of DTN. Section 3 explains about the different credit based incentive mechanisms of DTN. Section 4 gives the comparative study of existing credit based incentive protocols and Section 5 discusses conclusion and further research issues.

\section{Selfish Nodes in DTN}

In a typical DTNs deployment, people from remote cities use their battery operated hand held devices like cell phones, PDAs. These devices are portable to carry, but they are energy constraint. In DTN to capture the node's behaviour in the DTNs context, Researcher struggled that cooperation was the willingness and ability of a node to participate in a bundle's delivery process. Again a node may not be self-cantered. And it was the limit of resources, for instance, the power, the buffer space and so on, forced the node not to help with others. The three issues of research interests' topic in the aspect of cooperative techniques [5]:

1. Investigate for the non-cooperative nodes.

2. The affections cooperation put on the performance of the network.

3. Design protocols for the peers' cooperation.

A lot of existing packet forwarding algorithms is based on the assumption that all the nodes are willing to forward messages for others but in the real applications of DTNs, such as social networks and Vehicular Ad Hoc Networks.

In order to increase their own rewards, the nodes may not be willing to forward messages under the constraint of energy. This rational behaviour is called selfish behaviour in DTNs. The presence of selfish nodes could cause catastrophic damage to any well-designed routing scheme and jeopardize the whole network. Then how to incite the selfish nodes to take part in the relay of messages has become an interesting and essential issue which has attracted many researchers focusing on this problem. The 


\section{International Journal of Science and Research (IJSR) \\ ISSN (Online): 2319-7064}

Index Copernicus Value (2013): 6.14 | Impact Factor (2015): 6.391

method to solve this problem is often called incentive mechanism.

\section{Credit Based Incentive Mechanisms}

As we discussed in Section 2, how to stimulate the nodes joining in the relaying of messages has become a hot topic during these years. And in this section, we will make a detailed analysis of the existing credit based incentive mechanisms.

\subsection{SMART Mechanism}

Haojin Zhu et al. [6] propose a SMART (Secure multilayer credit based incentive) scheme. SMART scheme enforces DTN nodes to relay bundles towards the destination node. The main aim of SMART is to gain cooperation between nodes in bundle forwarding. Also, SMART overcomes various attacks in DTN.

SMART is based on the notion of naive multilayer coin which provides electronic credit to nodes for forwarding bundles towards the destination node. When a node in DTN sends a bundle, the sending node will lose credit and other intermediate node gets credit for their help and cooperation. In SMART, the first layer also called base layer is generated by source node which indicates the credit value and reward policies. In bundle relaying to the destination each intermediate node will generate a new layer called endorsed layer based on previous layers by appending digital signature. Layered coins are used for tracking the successful data delivery process. Each intermediate node in route periodically submits its collected coins to the centralised virtual bank. This virtual bank calculates credit associated with each intermediate node and charges the bundle sender nodes.

\subsection{Pi Mechanism}

A Practical incentive protocol $(\mathrm{Pi})$ is proposed by Rongxing $\mathrm{Lu}$ et al[7]. Pi protocol handles the selfish behaviour of a node in DTN. In message relaying of DTN when source node forwards a bundle to the destination node through intermediate nodes, source attaches some incentive or credit to the bundle which is fair and attractive. In PI, fair incentive mechanism is used to stimulate the nodes to participate in bundle forwarding to destination and also achieved better packet delivery ratio in DTN by using this protocol. Pi protocol will resist to attacks initiated by the selfish node which are Layer Adding Attack, Layer Removing attack and free ride attack.

In Pi protocol, there exists trusted third party i.e., trusted Authority (TA). The Role of TA is to perform clearance of credit and reputation of a DTN node and each node has to register itself to TA before joining DTN routing. In initialization phase, each node obtains its personal credit account (PCA) and personal reputation account (PRA) from TA. DTN nodes report to TA for clearance of credit and reputation when they get in touch with nearby TA. For example, in vehicular network vehicular nodes gets in touch with roadside TA for their reputation and clearance.
In SMART intermediate nodes get reputation and credit values for successful delivery of bundle to destination however for unsuccessful delivery nodes will also gets only reputation value hence PI protocol is Fair protocol.

\subsection{IPAD Mechanism}

An incentive and privacy-aware data dissemination (IPAD) strategy proposed by Rongxing Lu et al. [9]. IPAD is used in opportunistic networks (OPPNETs) and describes how to protect mobile nodes'

\section{Location privacy \\ 2. Identity privacy \\ 3. Social profile privacy}

The Advantage of IPAD scheme is to address above stated challenges and provide a secure incentive for privacyaware data dissemination. In IPAD, each node has a family of pseudo IDs and periodically changes its current pseudo ID to preserve owns privacy. When a bundle originating node sends a time-valuable data to its group of friends, it attaches an incentive with the bundles. This attached incentive with bundle stimulates the selfish nodes to help or cooperate in bundle forwarding process and improve the bundle delivery ratio and average time in opportunistic networks.

\subsection{TIS Mechanism}

Jun Zhou et al.[10] propose a TIS mechanism. TIS is a Threshold credit-based mechanism and based on the modified model of population dynamics. TIS enforce and maximize the vehicular nodes' interest and cooperation among the DTN intermediate nodes. TIS gives each node to equal opportunity in packet forwarding and achieves the fairness in credits offered to nodes in the network. TIS also address the layer adding attack raised due to the collusion of intermediate vehicular nodes. In vehicular DTN,

\section{High probability}

2. High Delivery ratio and

3. Low average delay is achieved in TIS mechanism.

\subsection{Win-Coupon Mechanism}

Xuejun Zhuo et al. [11] propose a Win-Coupon Strategy. Win-Coupon mechanism uses the concept of reverse auctioning for incentive mechanism to stimulate users to help cellular traffic offloading. In this mechanism role of the buyer is played by network operator who provides coupons to users. The coupon offered by the buyer is a cost to users to wait for some time and periodically offload the cellular traffic. The User sends bids along with request message to the buyer i.e., network operator. Each bid coming from the user towards network operator contains information about how long user is willing to wait for relaying messages and number of coupon he wants in return for the waiting of extra delay. Using bid info the network operator judge users' for the extra delay and also users' offloading capacity should be taken into consideration in deciding the auction outcome. By using historical system parameters collected like mobility

\section{Volume 5 Issue 6, June 2016 www.ijsr.net}




\section{International Journal of Science and Research (IJSR) \\ ISSN (Online): 2319-7064}

Index Copernicus Value (2013): 6.14 | Impact Factor (2015): 6.391

patterns and users' data access node's future value of offloading is predicted by network modelling. The optimal auction outcome from bidding is to minimise the incentive cost of network operator subject to a given traffic offloading target according to bidder's offloading potential and delay tolerance. There are two steps in the auction, first allocation and second pricing step. In the Allocation step, the buyer decides the winning bidders and the waiting time of them. In pricing step, the buyer decides the cost of each winner and in final step, the buyer returns the bidder with auction result containing assigned delay and value of coupon to each bidder. The winning bidders are ready to receive the bundle via cellular network as they have obtained the coupon after the committed delay is reached in communication.

\subsection{COUPONS Mechanism}

Garyfalos et al. [12] propose the Coupon Mechanism. The Coupon mechanism is totally different from conventional incentive strategies because it does not address the selfishness problem in DTN by providing incentives in its basic form like reputation or credit value as most other schemes do but it does from an application scenario perspective. In coupon incentive is in a contextual form based on an ordered list of unique IDs [13] and this unique ID is attached to messages. Coupons initiate cooperation at the application layer. In coupons data sharing among nodes is through opportunistic contact. Coupon scheme is designed to have content sharing by stimulating adaptive interactions between potential users. By basic flooding mechanism, coupons achieve the data dissemination, in this bundles are continuously broadcast as a node/user moves which are assumed to be independent and gets in contact with another users. A positive acknowledge from contacted user initiates the transmission of coupon via preconfigured user profiles or a three-way handshake based pull model. In coupon, users are stimulated in relaying their messages in pyramid manner i.e., up to the destination. In coupons scheme, every independent user/node is associated with unique ID and shares a coupon to the neighbour node to which node is in contact, building an ordered list of IDs appended with message. This ordered unique IDs associated with each intermediate node decides the path for forwarding bundles towards the destination. If node is participating in successful bundle delivery to the destination gets reward in the form of credit. Credit is nothing but a coupon in Coupon scheme. The nodes residing at the top of the pyramid gets more credit than the nodes which are residing at the bottom of pyramid. Coupons also handle the attacks initiated by malicious nodes such as obtaining more credit by deviating defined mechanism and unbalance the system by exploiting algorithm's weakness.

\section{Comparison}

In section-Credit Based Incentive Mechanisms, we have surveyed and analysed credit based incentive mechanisms. In this section, we have stated comparison of credit based incentive mechanisms as shown in table 1 .

From comparison, we can conclude the below points.

1. Most of the credit based mechanisms are depends on trusted third party.

2. The main reason of the selfishness behaviour of a node is the Limited resources of DTN node such as battery power and limited storage buffer.

Table 1: Comparison of Incentive Schemes

\begin{tabular}{|c|c|c|c|}
\hline $\begin{array}{l}\text { Credit Based } \\
\text { Incentive } \\
\text { Mechanisms }\end{array}$ & Basic Theme & Advantages & Disadvantages \\
\hline SMART [6] & $\begin{array}{l}\text { 1. Single copy (unicast) and Multi-copy (multicast) data } \\
\text { forwarding } \\
\text { 2. Layered coin provides virtual electronic credit as an } \\
\text { incentive }\end{array}$ & Scalability is very high & $\begin{array}{l}\text { 1.Require trusted third } \\
\text { party } \\
\text { 2.Digital signature as } \\
\text { aggregation has chain signature } \\
\text { communication } \\
\text { overhead }\end{array}$ \\
\hline Pi [7] & $\begin{array}{l}\text { 1. Combined approach of credit and reputation for incentive } \\
\text { provision. } \\
\text { 2. For successful packet delivery to destination, } \\
\text { intermediates helping nodes gets credit as well as reputation } \\
\text { 3. For unsuccessful packet delivery to destination, } \\
\text { intermediate helping nodes only gets reputation from trusted } \\
\text { third party }\end{array}$ & $\begin{array}{l}\text { It address the anti-black hole } \\
\text { Attack }\end{array}$ & $\begin{array}{l}\text { 1.Require trusted third } \\
\text { party } \\
\text { 2.No multi-copy } \\
\text { Forwarding }\end{array}$ \\
\hline IPAD [9] & $\begin{array}{l}\text { Secure Incentive strategy which is fair and with protection of } \\
\text { mobile nodes' privacy for efficiently disseminating a time- } \\
\text { valuable data }\end{array}$ & $\begin{array}{l}\text { It provides mobile nodes: } \\
\text { 1. Identity privacy } \\
\text { 2. Location } \\
\text { privacy\& } \\
\text { 3. Social profile } \\
\text { Privacy }\end{array}$ & $\begin{array}{l}\text { Require trusted third } \\
\text { Party }\end{array}$ \\
\hline TIS [10] & $\begin{array}{l}\text { Use of modified population dynamics model as the basis of } \\
\text { Incentive scheme }\end{array}$ & $\begin{array}{l}\text { It address the resisting the Layer } \\
\text { adding attack }\end{array}$ & $\begin{array}{l}\text { Require trusted third } \\
\text { Party }\end{array}$ \\
\hline $\begin{array}{l}\text { Win-Coupon } \\
{[11]}\end{array}$ & $\begin{array}{l}\text { DTN Nodes which helps in forwarding the bundles gets } \\
\text { currency as Incentive and } \\
\text { nodes sending bundles for themselves loose } \\
\text { currency }\end{array}$ & $\begin{array}{l}\text { It address the anti-hiding } \\
\text { Attack }\end{array}$ & $\begin{array}{l}\text { Require trusted third } \\
\text { Party }\end{array}$ \\
\hline $\begin{array}{l}\text { Coupons [12] } \\
\text { and [13] }\end{array}$ & $\begin{array}{l}\text { Incentive is contextual and not like conventional incentive } \\
\text { and is based on an } \\
\text { ordered list of unique IDs appended to messages }\end{array}$ & $\begin{array}{l}\text { Scalability is very } \\
\text { High }\end{array}$ & $\begin{array}{l}\text { Charging rate is } \\
\text { application dependant }\end{array}$ \\
\hline
\end{tabular}

Volume 5 Issue 6, June 2016 www.ijsr.net 


\section{International Journal of Science and Research (IJSR) \\ ISSN (Online): 2319-7064}

Index Copernicus Value (2013): 6.14 | Impact Factor (2015): 6.391

\section{Conclusion}

In this paper, we surveyed different credit based incentive mechanisms and analysis of these schemes based on their basic theme, advantages and disadvantages is summarized. In DTN, selfish behaviour of a node is the main issue and is addressed through various credit based incentive schemes but still DTN requires more research to address the selfishness problem of a node so that selfish nodes will also cooperate and participate in packet delivery to destination and increase the packet delivery ratio. A part of the selfish behaviour of a node, there are many issues in DTN as below:

1. Handling replays

2. Routing protocol security

3. Key management

4. Traffic analysis

5. Performance issues

6. Multicast security

\section{References}

[1] F. Warthman, Delay-Tolerant Networks (DTNs) A Tutorial, http://www.dtnrg.org/docs/tutorials/warthman-1.1.pdf

[2] Alex (Sandy) Pentland, Richard Fletcher and Amir Hasson, 'DakNet: Rethinking Connectivity in Developing Nations', Published by the IEEE Computer Society.

[3] K. Scott, S. Burleigh, Bundle protocol specification, http://www.rfceditor. org/rfc/pdfrfc/rfc5050.txt.pdf

[4] V. Cerf, S. Burleigh, A. Hooke, L. Torgerson, R. Durst, K. Scott, K. Fall, H. Weiss, Delay-Tolerant Networking Architecture, http://www.rfceditor.org/rfc/pdfrfc/rfc4838.txt.pdf

[5] Xin Jiang, Xiang-Yu Bai, 'A Survey on Incentive Mechanism of Delay Tolerant Networks', (C) 2013 IEEE.

[6] Haojin Zhu, Xiaodong Lin, Me, Rongxing Lu, Yanfei Fan, and Xuemin (Sherman) Shen,' SMART: A Secure Multilayer Credit-Based Incentive Scheme for Delay-Tolerant Networks', IEEE Transaction on Vehicular Technology, Vol. 58, No. 8, October 2009.

[7] Rongxing Lu, Xiaodong Lin, Haojin Zhu, Xuemin (Sherman) Shen, Bruno Preiss, 'Pi: A Practical Incentive Protocol for Delay Tolerant Networks', IEEE Transactions on Wireless Communications, Vol. 9, NO. 4, April 2010.

[8] Chen B, Chan M. MobiCent: A credit-based incentive system for disruption tolerant networks [C] //Proc of 29th Conference on Computer Communications (INFOCOM'10). Piscataway, NJ: IEEE, 2010: 875883.

[9] Rongxing Lu, Xiaodong Lin, Zhiguo Shi, Bin Cao, Xuemin (Sherman) Shen, 'IPAD: An Incentive \& Privacy-Aware Data Dissemination Scheme in Opportunistic Networks', 2013 Proceedings IEEE INFOCOM.

[10]Jun Zhou, Zhenfu Cao, 'TIS: A Threshold Incentive Scheme for Secure and Reliable Data Forwarding in Vehicular Delay Tolerant Networks', GLOBECOM
2012 - Communication And Information System Security Symposium.

[11] Xuejun Zhuo, Wei Gao, Guohong Cao \& Sha Hua, 'An Incentive Framework for Cellular Traffic Offloading', IEEE Transactions On Mobile Computing, Vol. 13, No. 3, March 2014.

[12]A. Garyfalos and K. C. Almeroth, 'Coupons: A multilevel incentive scheme for information dissemination in mobile networks', IEEE Transactions on Mobile Computing, 7(6): June 2008, pp. 792-804.

[13] Mieso K. Denko, Mobile Opportunistic Networks Architectures, Protocols and Applications. CRC Press, 2011

Volume 5 Issue 6, June 2016 www.ijsr.net 